\title{
Monophosphaporphyrins: Oxidative $\pi$-Extension at the Peripherally-Fused Carbocycle of the Phosphaporphyrin Ring
}

Yoshihiro Matano, ${ }^{* \dagger}$ Makoto Nakashima,${ }^{\dagger}$ Takashi Nakabuchi, ${ }^{\dagger}$ Hiroshi Imahori, ${ }^{\dagger,}$ Shinya Fujishige, ${ }^{\S}$ and Haruyuki Nakano ${ }^{\S}$

${ }^{\dagger}$ Department of Molecular Engineering, Graduate School of Engineering, Kyoto University, Nishikyo-ku, Kyoto 615-8510, Japan, "Institute for Integrated Cell-Material Sciences, Kyoto University, Nishikyo-ku, Kyoto 615-8510, Japan, and ${ }^{\S}$ Department of Chemistry, Graduate School of Sciences, Kyushu University, Fukuoka 812-8581, Japan

${ }^{\dagger}$ Graduate School of Engineering, Kyoto University. ${ }^{\ddagger}$ Institute for Integrated Cell-Material Sciences, Kyoto University. ${ }^{\S}$ Kyushu University.

Email:matano@scl.kyoto-u.ac.jp FAX: $+81-75-383-2571$

\section{Contents:}

General:

Synthesis and Characterization of 5-9:

SI-2

Density Functional Theory (DFT) Calculations on Model Compounds: $\quad$ SI-6

Figures S1 S10:

SI-8 


\section{General}

${ }^{1} \mathrm{H},{ }^{13} \mathrm{C}\left\{{ }^{1} \mathrm{H}\right\}$, and ${ }^{31} \mathrm{P}\left\{{ }^{1} \mathrm{H}\right\}$ NMR spectra were measured in $\mathrm{CDCl}_{3}$ or $\mathrm{CD}_{2} \mathrm{Cl}_{2}$. Chemical shifts are reported as the relative value vs. tetramethylsilane $\left({ }^{1} \mathrm{H},{ }^{13} \mathrm{C}\right)$ and $\mathrm{H}_{3} \mathrm{PO}_{4}\left({ }^{31} \mathrm{P}\right)$, respectively. MALDI-TOF and HR-FAB-MS mass spectra were measured using CHCA and 3-nitrobenzyl alcohol as matrices, respectively. Electrochemical measurements were performed using a glassy carbon working electrode, a platinum wire counter electrode, and an $\mathrm{Ag} / \mathrm{Ag}^{+}\left[0.01 \mathrm{M} \mathrm{AgNO}_{3}, 0.1 \mathrm{M}\right.$ $\left.n \mathrm{Bu}_{4} \mathrm{NPF}_{6}(\mathrm{MeCN})\right]$ reference electrode. The potentials were calibrated with ferrocenium/ferrocene $\left[E_{\text {mid }}=+0.20 \mathrm{~V}\right.$ vs Ag/AgNO 3 . Dichloromethane $\left(\mathrm{CH}_{2} \mathrm{Cl}_{2}\right)$ and toluene and THF were distilled from $\mathrm{CaH}_{2}\left(\mathrm{CH}_{2} \mathrm{Cl}_{2}\right.$, toluene) or sodium benzophenone ketyl (THF) before use. The phosphatripyrrane $\mathbf{3}^{1}$ and 2,5-dibenzoylpyrrole ${ }^{2}$ were prepared according to the reported procedure. Other chemicals and solvents were of reagent grade quality, purchased commercially and used without further purification unless otherwise noted. Thin-layer chromatography was performed with Alt. 5554 DC-Alufolien Kieselgel $60 \mathrm{~F}_{254}$ (Merck). Gravity column chromatography was carried out using Silica gel or Alumina in open air.

\section{Synthesis and Characterization of 5-9}

$\sigma^{4}$-Porphyrinogen 5: $\mathrm{NaBH}_{4}(720 \mathrm{mg}, 18.9 \mathrm{mmol})$ was added to a solution of 2,5-dibenzoylpyrrole (110 mg, $0.39 \mathrm{mmol}$ ) in THF/MeOH (total $24 \mathrm{~mL} ; \mathrm{v} / \mathrm{v}=3 / 1$ ), which was then stirred for $30 \mathrm{~min}$ at room temperature. After adding a mixture of water $(10 \mathrm{~mL})$ and $\mathrm{CH}_{2} \mathrm{Cl}_{2}(30 \mathrm{~mL})$, the organic phase was separated, dried over $\mathrm{K}_{2} \mathrm{CO}_{3}$, and evaporated. The crude product 4 was dissolved in $\mathrm{CH}_{2} \mathrm{Cl}_{2}$ solution (120 mL) containing $\mathbf{3}$ (120 mg. $0.30 \mathrm{mmol}$ ), followed by addition of $\mathrm{BF}_{3} \cdot \mathrm{OEt}_{2}$ ( $39 \mu \mathrm{L}$, $0.30 \mathrm{mmol})$. After $5 \mathrm{~min}$ of stirring at room temperature, $\mathrm{Et}_{3} \mathrm{~N}(75 \mu \mathrm{L}, 0.54 \mathrm{mmol})$ was added and the

\footnotetext{
1 Matano, Y.; Nakabuchi, T.; Miyajima, T.; Imahori, H.; Nakano, H. Org. Lett. 2006, 8, 5713. 2 (a) Degani, I.; Fochi, R. J. Chem. Soc. Perkin Trans. 1 1976, 323. (b) Cadamuro, S.; Degani, I.; Fochi, R.; Gatti, A.; Regondi, V. Synthesis 1987, 311. (c) Barbero, M.; Cadamuro, S.; Degani, I.; Fochi, R.; Gatti, A.; Regondi, V. J. Org. Chem. 1988, 53, 2245.
} 
resulting mixture was washed with distilled water $(50 \mathrm{~mL})$, dried over $\mathrm{Na}_{2} \mathrm{SO}_{4}$, and evaporated. The crude product was subjected to silica gel column chromatography $\left(\mathrm{CH}_{2} \mathrm{Cl}_{2} /\right.$ hexane $\left.=2 / 1\right)$ to give $\mathbf{5}$ as a mixture of three diastereomers $\left(R_{\mathrm{f}}=0.5-0.6 ; 82 \mathrm{mg}, 43 \%\right)$. The ${ }^{1} \mathrm{H}$ and ${ }^{31} \mathrm{P}$ NMR spectra indicated that three major diastereomers $(\mathbf{A}, \mathbf{B}, \mathbf{C})$ were included in a ratio of 10:5:4. ${ }^{1} \mathrm{H} \mathrm{NMR}\left(400 \mathrm{MHz}, \mathrm{CDCl}_{3}\right.$, $\left.25^{\circ} \mathrm{C}\right)$ : Diastereomer A: $\delta 1.92-2.00(\mathrm{~m}, 1 \mathrm{H}), 2.06-2.14(\mathrm{~m}, 1 \mathrm{H}), 2.31-2.43(\mathrm{~m}, 4 \mathrm{H}), 3.42-3.50(\mathrm{~m}$, 4H), $5.25(\mathrm{~s}, 1 \mathrm{H}), 5.40(\mathrm{~s}, 1 \mathrm{H}), 5.47-5.48(\mathrm{~m}, 1 \mathrm{H}), 5.53-5.54(\mathrm{~m}, 1 \mathrm{H}), 5.72-5.73(\mathrm{~m}, 1 \mathrm{H}), 5.81-5.82$ $(\mathrm{m}, 1 \mathrm{H}), 5.87-5.88(\mathrm{~m}, 1 \mathrm{H}), 6.09(\mathrm{dd}, J=2.9 \mathrm{~Hz}, J=2.9 \mathrm{~Hz}, 1 \mathrm{H}), 7.11-7.37(\mathrm{~m}, 10 \mathrm{H}), 7.47-7.54(\mathrm{~m}$, 3H), 7.67 (br-s, 1H), 7.83-7.89 (m, 2H), 9.64 (br-s, 1H), 10.10 (br-s, 1H); Diastereomer B:

$\delta 1.86-1.93(\mathrm{~m}, 1 \mathrm{H}), 2.06-2.14(\mathrm{~m}, 1 \mathrm{H}), 2.31-2.39(\mathrm{~m}, 4 \mathrm{H}), 3.42-3.50(\mathrm{~m}, 4 \mathrm{H}), 5.41-5.42(\mathrm{~m}, 4 \mathrm{H})$, 5.82-5.83 (m, 2H), $6.16(\mathrm{~d}, J=2.4 \mathrm{~Hz}, 2 \mathrm{H}), 7.12-7.40(\mathrm{~m}, 10 \mathrm{H}), 7.45-7.54(\mathrm{~m}, 3 \mathrm{H}), 7.67(\mathrm{br}-\mathrm{s}, 1 \mathrm{H})$, 7.82-7.87 (m, 2H), 9.64 (br-s, 2H); Diastereomer C: $\delta$ 2.02-2.09 (m, 2H), 2.30-2.46 (m, 4H), 3.43-3.47 (m, 4H), $5.36(\mathrm{~s}, 2 \mathrm{H}), 5.51(\mathrm{~d}, J=2.4 \mathrm{~Hz}, 2 \mathrm{H}), 5.75-5.76(\mathrm{~m}, 2 \mathrm{H}), 5.83-5.84(\mathrm{~m}, 2 \mathrm{H})$, 7.13-7.30 (m, 10H), 7.44-7.55 (m, 3H), 7.83-7.87 (m, 2H), 8.05 (br-s, 1H), 9.99 (br-s, 2H); ${ }^{31} \mathrm{P}\left\{{ }^{1} \mathrm{H}\right\}$ NMR (162MHz, $\left.\mathrm{CDCl}_{3}, 25^{\circ} \mathrm{C}\right): \delta 70.4,71.2,72.0$; MS (MALDI-TOF): $m / z 633\left(\mathrm{M}^{+}\right)$.

$\boldsymbol{\sigma}^{3}$-Phosphaporphyrinogen 6: To a toluene solution $(10 \mathrm{~mL})$ of $5(135 \mathrm{mg}, 0.21 \mathrm{mmol})$ was added $\mathrm{P}\left(\mathrm{NMe}_{2}\right)_{3}(0.25 \mathrm{~mL}, 1.4 \mathrm{mmol})$, and the mixture was then stirred under reflux for $40 \mathrm{~h}$. The resulting mixture was concentrated under reduced pressure and subjected to silica gel column chromatography (hexane $\left./ \mathrm{CH}_{2} \mathrm{Cl}_{2}=1 / 1\right)$ to give 6 as a mixture of diastereomers $\left(R_{\mathrm{f}}=0.4-0.5 ; 120 \mathrm{mg}, 95 \%\right)$ : The ratio of the three diastereomers (A, B, C) was found to be 10:4:4. ${ }^{1} \mathrm{H} \mathrm{NMR}\left(400 \mathrm{MHz}, \mathrm{CDCl}_{3}, 25^{\circ} \mathrm{C}\right)$ : Diastereomer A: $\delta 2.12-2.16(\mathrm{~m}, 2 \mathrm{H}), 2.22-2.34(\mathrm{~m}, 2 \mathrm{H}), 2.51-2.59(\mathrm{~m}, 2 \mathrm{H}), 3.34-3.42(\mathrm{~m}, 2 \mathrm{H})$, 3.62-3.75 (m, 2H), $5.33(\mathrm{~s}, 1 \mathrm{H}), 5.41(\mathrm{~s}, 1 \mathrm{H}), 5.65-5.66(\mathrm{~m}, 1 \mathrm{H}), 5.68-5.69(\mathrm{~m}, 1 \mathrm{H}), 5.79-5.80(\mathrm{~m}$, 1H), 5.84-5.85 (m, 2H), 5.99-6.00 (m, 1H), 7.14-7.33 (m, 15H), 7.79 (br-s, 1H), 8.23 (br-s, 1H), 8.35 (br-s, 1H); Diastereomer B: $\delta$ 2.19-2.28 (m, 2H), 2.35-2.39 (m, 2H), 2.57-2.62 (m, 2H), 3.39-3.43 (m, 2H), 3.68-3.77 (m, 2H), $5.40(\mathrm{~s}, 2 \mathrm{H}), 5.73-5.74(\mathrm{~m}, 2 \mathrm{H}), 5.78-5.80(\mathrm{~m}, 4 \mathrm{H}), 7.14-7.32(\mathrm{~m}, 15 \mathrm{H}), 7.81$ (br-s, 1H), 7.98 (br-s, 2H); Diastereomer C: $\delta 2.22(\mathrm{~m}, 4 \mathrm{H}), 2.54(\mathrm{~m}, 2 \mathrm{H}), 3.35-3.42(\mathrm{~m}, 2 \mathrm{H})$,

$$
\text { SI- } 3
$$


3.62-3.75 (m, 2H), $5.39(\mathrm{~s}, 2 \mathrm{H}), 5.67-5.69(\mathrm{~m}, 2 \mathrm{H}), 5.79-5.80(\mathrm{~m}, 2 \mathrm{H}), 5.83-5.84(\mathrm{~m}, 2 \mathrm{H}), 7.14-7.33$ (m, 15H), 7.94 (br-s, 1H), 8.32 (br-s, $2 \mathrm{H}) ;{ }^{31} \mathrm{P}\left\{{ }^{1} \mathrm{H}\right\} \mathrm{NMR}\left(162 \mathrm{MHz}, \mathrm{CDCl}_{3}, 25{ }^{\circ} \mathrm{C}\right): \delta 30.4,32.5,32.7$; MS (MALDI-TOF): $m / z, 601\left(\mathrm{M}^{+}\right)$.

7 and 8: To a solution of 6 (25 mg, $0.042 \mathrm{mmol})$ in degassed toluene (4 mL), was added a toluene (2 $\mathrm{mL}$ ) solution of DDQ (31 mg, $0.14 \mathrm{mmol}$ ) over $15 \mathrm{~min}$ at room temperature. After stirred for $30 \mathrm{~min}$, the reaction mixture was filtered through a pad of alumina $\left(2 \mathrm{~cm} \mathrm{dia} \times 1.5 \mathrm{~cm}, \mathrm{CH}_{2} \mathrm{Cl}_{2}\right.$, then EtOAc). Further purification of the crude products by alumina column chromatography, followed by recrystallization from $\mathrm{CH}_{2} \mathrm{Cl}_{2}$ /hexane, afforded 7 (hexane/EtOAc $=15 / 1, R_{\mathrm{f}}=0.6$ ) as a reddish purple solid (4.2 $\mathrm{mg}, 17 \%)$ and $\mathbf{8}$ (hexane/EtOAc $\left.=15 / 1, R_{\mathrm{f}}=0.3\right)$ as a dark-purple solid (2.0 mg, 8\%). 18 $\pi-\sigma^{3}$-Phosphaporphyrin 7: ${ }^{1} \mathrm{H}$ NMR $\left(400 \mathrm{MHz}, \mathrm{CDCl}_{3}, 25^{\circ} \mathrm{C}\right): \delta-0.59$ (br-s, $\left.1 \mathrm{H}\right), 2.43(\mathrm{dd}, J=$ $\left.7.3 \mathrm{~Hz},{ }^{3} J_{\mathrm{P}, \mathrm{H}}=4.4 \mathrm{~Hz}, 2 \mathrm{H}\right), 3.06(\mathrm{~m}, 1 \mathrm{H}), 3.33(\mathrm{~m}, 1 \mathrm{H}), 3.83(\mathrm{~m}, 2 \mathrm{H}), 4.53(\mathrm{~m}, 2 \mathrm{H}), 5.23(\mathrm{dd}, J=7.3$ $\mathrm{Hz}, 7.3 \mathrm{~Hz}, 2 \mathrm{H}), 5.68$ (t, $J=7.3 \mathrm{~Hz}, 1 \mathrm{H}), 7.69(\mathrm{~m}, 6 \mathrm{H}), 7.97(\mathrm{~d}, J=7.3 \mathrm{~Hz}, 2 \mathrm{H}), 8.24(\mathrm{~m}, 2 \mathrm{H}), 8.35(\mathrm{~d}$, $J=4.4 \mathrm{~Hz}, 2 \mathrm{H}), 8.62(\mathrm{~d}, J=2.0 \mathrm{~Hz}, 2 \mathrm{H}), 8.67(\mathrm{~d}, J=4.4 \mathrm{~Hz}, 2 \mathrm{H}), 10.18\left(\mathrm{~d},{ }^{3} J_{\mathrm{P}, \mathrm{H}}=16.1 \mathrm{~Hz}, 2 \mathrm{H}\right)$; ${ }^{13} \mathrm{C}\left\{{ }^{1} \mathrm{H}\right\}$ NMR $\left(100 \mathrm{MHz}, \mathrm{CDCl}_{3}, 25^{\circ} \mathrm{C}\right): \delta 28.78$ (s), $30.30(\mathrm{~s}), 120.61(\mathrm{~d}, J=4.8 \mathrm{~Hz}), 124.77(\mathrm{~d}, J=$ $4.8 \mathrm{~Hz}$ ), 125.03 (s), 125.73 (s), 126.49 (s), 127.70 (d, $J=16.3 \mathrm{~Hz}), 127.99$ (d, J = 9.6 Hz), 131.45 (s), 132.53, (s), 134.15 (s), 134.56 (s), 135.97 (s), 137.55 (s), 142.60 (s), 149.06 (d, J = $16.3 \mathrm{~Hz}), 153.29$ (s), $159.36(\mathrm{~d}, J=3.8 \mathrm{~Hz}) ;{ }^{31} \mathrm{P}\left\{{ }^{1} \mathrm{H}\right\}$ NMR $\left(162 \mathrm{MHz}, \mathrm{CDCl}_{3}, 25^{\circ} \mathrm{C}\right): \delta-5.2 ; \mathrm{UV} / \mathrm{Vis}\left(\mathrm{CH}_{2} \mathrm{Cl}_{2}\right): \lambda_{\max }(\varepsilon)$ 431 (137000), 486 (8600), 522 (9000), 555 (6100), 636 (2000), $701 \mathrm{~nm}(3100) ;$ MS (FAB): $m / z=596$ $[M+\mathrm{H}]^{+}$; HR-FAB-MS: Calcd for $\mathrm{C}_{41} \mathrm{H}_{30} \mathrm{~N}_{3} \mathrm{P}\left[M^{+}\right]$, 595.2177; Found, 595.2182. In the ${ }^{13} \mathrm{C}\left\{{ }^{1} \mathrm{H}\right\} \mathrm{NMR}$ spectrum, one carbon peak could not be detected.

22 $\pi-\sigma^{4}$-Phosphaporphyrin 8: ${ }^{1} \mathrm{H}$ NMR $\left(400 \mathrm{MHz}, \mathrm{CDCl}_{3}, 25^{\circ} \mathrm{C}\right): \delta 5.40(\mathrm{br}-\mathrm{s}, 2 \mathrm{H}), 5.60(\mathrm{dd}, J=7.3$ $\left.\mathrm{Hz},{ }^{3} J_{\mathrm{P}, \mathrm{H}}=12.2 \mathrm{~Hz}, 2 \mathrm{H}\right), 6.60(\mathrm{~m}, 2 \mathrm{H}), 6.92(\mathrm{t}, J=7.6 \mathrm{~Hz}, 1 \mathrm{H}), 7.68(\mathrm{~m}, 8 \mathrm{H}), 7.94(\mathrm{~s}, 2 \mathrm{H}), 8.02(\mathrm{~d}, J=$ $4.4 \mathrm{~Hz}, 2 \mathrm{H}), 8.16(\mathrm{~d}, J=3.9 \mathrm{~Hz}, 2 \mathrm{H}), 8.19(\mathrm{~d}, J=4.4 \mathrm{~Hz}, 2 \mathrm{H}), 8.25(\mathrm{~d}, J=7.3 \mathrm{~Hz}, 2 \mathrm{H}), 9.06(\mathrm{dt}, J=$ $\left.3.9 \mathrm{~Hz},{ }^{5} \mathrm{~J}_{\mathrm{P}, \mathrm{H}}=5.4 \mathrm{~Hz}, 1 \mathrm{H}\right), 9.21\left(\mathrm{~d},{ }^{3} J_{\mathrm{P}, \mathrm{H}}=35.6 \mathrm{~Hz}, 2 \mathrm{H}\right) ;{ }^{13} \mathrm{C}\left\{{ }^{1} \mathrm{H}\right\} \mathrm{NMR}\left(100 \mathrm{MHz}, \mathrm{CDCl}_{3}, 25^{\circ} \mathrm{C}\right): \delta$ $106.29(\mathrm{~d}, J=12.5 \mathrm{~Hz}), 112.09(\mathrm{~d}, J=7.7 \mathrm{~Hz}), 125.13$ (d, $J=24.9 \mathrm{~Hz}), 125.93(\mathrm{~s}), 126.91(\mathrm{~s}), 127.55$ 
$(\mathrm{d}, J=12.5 \mathrm{~Hz}), 128.03(\mathrm{~s}), 128.29(\mathrm{~s}), 129.26(\mathrm{~d}, J=9.9 \mathrm{~Hz}), 130.98(\mathrm{~s}), 131.39(\mathrm{~s}), 133.47(\mathrm{~d}, J=$ $9.6 \mathrm{~Hz}), 135.00$ (s), 137.82 (s), 141.82 (s), 142.25 (s), 143.17 (s), 143.70 (s), 146.07 (d, J = $7.7 \mathrm{~Hz}$ ), $156.34(\mathrm{~s}) ;{ }^{31} \mathrm{P}\left\{{ }^{1} \mathrm{H}\right\}$ NMR (162MHz, $\left.\mathrm{CDCl}_{3}, 25^{\circ} \mathrm{C}\right): \delta 28.6$; UV/Vis $\left(\mathrm{CH}_{2} \mathrm{Cl}_{2}\right): \lambda_{\max }(\varepsilon) 422(42100)$, 494 (77200), 649 (9100), 708 nm (11400); MS (FAB): m/z 610 [M+H] ${ }^{+}$; HR-FAB-MS: Calcd for $\mathrm{C}_{41} \mathrm{H}_{28} \mathrm{~N}_{3} \mathrm{OP}\left[M^{+}\right], 609.1970 ;$ Found, 609.1975.

$\sigma^{4}$-Phosphaporphyrinogen 9: $\mathrm{A} \mathrm{CH}_{2} \mathrm{Cl}_{2}$ solution $(6 \mathrm{~mL})$ of $m$-chloroperbenzoic acid (29 $\mathrm{mg}, 0.13$ mmol) was added dropwise at $-30{ }^{\circ} \mathrm{C}$ to a solution of $6(61 \mathrm{mg}, 0.10 \mathrm{mmol})$ in $9 \mathrm{~mL}$ of $\mathrm{CH}_{2} \mathrm{Cl}_{2}$. The mixture was stirred at $0{ }^{\circ} \mathrm{C}$ for $1 \mathrm{~h}$ and then concentrated under reduced pressure. The solid residue was subjected to silica-gel column chromatography $\left(\mathrm{CH}_{2} \mathrm{Cl}_{2} /\right.$ hexane $\left.=1 / 1\right)$ to give 9 as a mixture of three diastereomers $\left(R_{\mathrm{f}}=0.3-0.4 ; 31 \mathrm{mg}, 50 \%\right)$. The ratio of the three diastereomers $(\mathbf{A}, \mathbf{B}, \mathbf{C})$ was found to be 10:4:3. ${ }^{1} \mathrm{H}$ NMR (400 $\left.\mathrm{MHz}, \mathrm{CDCl}_{3}, 25^{\circ} \mathrm{C}\right)$ : Diastereomer $\mathbf{A}: \delta 1.92-2.00(\mathrm{~m}, 1 \mathrm{H})$, 2.02-2.11 (m, 1H), 2.43-2.54 (m, 4H), 3.35-3.63 (m, 4H), $5.32(\mathrm{~s}, 1 \mathrm{H}), 5.42(\mathrm{~s}, 1 \mathrm{H})$, 5.49-5.51 (m, $1 \mathrm{H}), 5.57(\mathrm{dd}, J=2.9 \mathrm{~Hz}, J=2.9 \mathrm{~Hz}, 1 \mathrm{H}), 5.76(\mathrm{dd}, J=2.9 \mathrm{~Hz}, J=2.9 \mathrm{~Hz}, 1 \mathrm{H}), 5.79(\mathrm{dd}, J=2.4 \mathrm{~Hz}$, $J=2.4 \mathrm{~Hz}, 1 \mathrm{H}), 5.90(\mathrm{dd}, J=2.4 \mathrm{~Hz}, J=2.4 \mathrm{~Hz}, 1 \mathrm{H}), 6.13(\mathrm{dd}, J=2.9 \mathrm{~Hz}, J=2.9 \mathrm{~Hz}, 1 \mathrm{H}), 7.10-7.34$ (m, 8H), 7.43-7.56 (m, 5H), 7.76-7.81 (m, 2H), 7.89 (br-s, 1H), 10.05 (br-s, 1H), 10.40 (br-s, 1H); Diastereomer B: $\delta 1.92-2.00(\mathrm{~m}, 1 \mathrm{H}), 2.02-2.11(\mathrm{~m}, 1 \mathrm{H}), 2.38-2.58(\mathrm{~m}, 4 \mathrm{H}), 3.38-3.62(\mathrm{~m}, 4 \mathrm{H}), 5.42$ (s, 2H), 5.69 (d, $J=2.4 \mathrm{~Hz}, 2 \mathrm{H}), 5.81-5.83(\mathrm{~m}, 4 \mathrm{H}), 7.13-7.26(\mathrm{~m}, 10 \mathrm{H}), 7.46-7.56(\mathrm{~m}, 3 \mathrm{H})$, 7.71-7.79 (m, 2H), 8.24 (br-s, 1H), 10.23 (br-s, 2H); Diastereomer C: $\delta 1.90-2.16(\mathrm{~m}, 2 \mathrm{H}), 2.35-2.46$ (m, 2H), 2.53-2.62 (m, 2H), 3.41-3.66 (m, 4H), $5.44(\mathrm{~s}, 2 \mathrm{H}), 5.46-5.47(\mathrm{~m}, 2 \mathrm{H}), 5.81-5.82(\mathrm{~m}, 2 \mathrm{H})$, $6.19(\mathrm{~d}, J=2.4 \mathrm{~Hz}, 2 \mathrm{H}), 7.16-7.30(\mathrm{~m}, 10 \mathrm{H}), 7.46-7.54(\mathrm{~m}, 3 \mathrm{H}), 7.71-7.80(\mathrm{~m}, 2 \mathrm{H}), 8.22(\mathrm{br}-\mathrm{s}, 1 \mathrm{H})$, 10.21 (br-s, $2 \mathrm{H}) ;{ }^{31} \mathrm{P}\left\{{ }^{1} \mathrm{H}\right\}$ NMR $\left(162 \mathrm{MHz}, \mathrm{CDCl}_{3}, 25^{\circ} \mathrm{C}\right): \delta 59.2,59.5,59.6$; MS (MALDI-TOF): $\mathrm{m} / z$ $617\left(\mathrm{M}^{+}\right)$

Synthesis of 8 from 9: To a solution of $9(12 \mathrm{mg}, 0.019 \mathrm{mmol})$ in degassed toluene $(3 \mathrm{~mL})$, was added a toluene $(2 \mathrm{~mL})$ solution of DDQ $(15 \mathrm{mg}, 0.064 \mathrm{mmol})$ over $15 \mathrm{~min}$ at room temperature. After stirred 
for $1 \mathrm{~h}$, the reaction mixture was filtered through a pad of alumina $\left(2 \mathrm{~cm}\right.$ dia $\times 1.5 \mathrm{~cm}, \mathrm{CH}_{2} \mathrm{Cl}_{2}$ then EtOAc). Further purification of the crude product by alumina column chromatography, followed by recrystallization from $\mathrm{CH}_{2} \mathrm{Cl}_{2} /$ hexane, afforded $\mathbf{8}$ as a dark-purple solid (3.1 mg, 26\%).

X-ray Crystallographic Analyses of 7 and 8: Single crystals suitable for X-ray analysis were grown from $\mathrm{CH}_{2} \mathrm{Cl}_{2}-\mathrm{MeOH}$ at room temperature. X-ray crystallographic measurements were made on a Rigaku Saturn CCD area detector with graphite monochromated Mo-Ko radiation $(0.71070 \AA)$ at $-150{ }^{\circ} \mathrm{C}$. The data were corrected for Lorentz and polarization effects. The structures were solved by using direct methods ${ }^{3}$ and refined by full-matrix least squares techniques against $F^{2}$ using SHELXL-97. ${ }^{4}$ The non-hydrogen atoms were refined anisotropically, and hydrogen atoms were refined using the rigid model.

Photosensitized Oxidation of 7 to 8: $\mathrm{A} \mathrm{CH}_{2} \mathrm{Cl}_{2}$ solution of $7\left(1.0 \times 10^{-5} \mathrm{M}\right)$ in a quartz cuvette capped with a Teflon-coated silicone septum was bubbled with oxygen for 10 min and then monitored by UV/vis absorption spectroscopy. The results are shown in Figure S2.

Density Functional Theory (DFT) Calculations on Model Compounds: The structures of $\mathbf{1 1}$ and 12, as models for $\mathbf{7}$ and $\mathbf{8}$, respectively, were optimized using density functional theory (DFT). The basis set used was 6-311G(d,p). ${ }^{5}$ The functionals of DFT was the Becke 1988 exchange and Lee-Yang-Parr correlation functionals (B3LYP). ${ }^{6}$ The nucleus independent chemical shift (NICS) values ${ }^{7}$ were

3 SIR92: Altomare, A.; Cascarano, G.; Giacovazzo, C.; Guagliardi, A.; Burla, M.; Polidori, G.; Camalli, M. J. Appl. Cryst. 1994, 27, 435.

${ }^{4}$ Sheldrick, G. M. SHELXL-97, University of Göttingen, Germany, 1997.

5 (a) Krishnan, R.; Binkley, J. S.; Seeger, R.; Pople, J. A. J. Chem. Phys. 1980, 72, 650. (b) McLean, A. D.; Chandler, G. S. J. Chem. Phys. 1980, 72, 5639.

6 (a) Becke, A. D. J. Chem. Phys. 1988, 98, 5648. (b) Lee, C.; Yang, W.; Parr, R. G. Phys. Rev. B 1988, 37, 785 .

$$
\text { SI- } 6
$$


calculated at the Hartree-Fock level with gauge-including atomic orbitals (GIAOs) at the DFT optimized structures. The basis set used in the NICS value computations was $6-31+\mathrm{G}(\mathrm{d}) .{ }^{8}$ The optimized structures are depicted in Figures S3(a) and S4(a), and the bond lengths of the optimized structures are shown in Figures S3(b) and S4(b). The molecular orbitals near in energy to HOMO and LUMO are also depicted in Figures S3(c) and S4(c). All the calculations were carried out using the Gaussian 03 suite of programs. ${ }^{9}$ In the optimized structure of 11, the P-phenyl ring is found to be parallel to the $\mathrm{P}-\mathrm{HN}$ axis. However, the difference in energy between the optimized structure and the structure in which the P-phenyl ring is rotated by 90 degrees (i.e., the P-phenyl ring is vertical to the $\mathrm{P}-\mathrm{NH}$ axis $)$ is negligible $\left(0.16 \mathrm{kcal} \mathrm{mol}^{-1}\right)$. Thus, the binding for the $\mathrm{P}-$ phenyl ring rotation is considered to be very weak.

7 Schleyer, P. v. R.; Maerker, C.; Dransfeld, A.; Jiao, H.; van Eikema Hommes, N. J. R. J. Am. Chem. Soc. 1996, 118, 6317.

8 (a) Hehre, W. J.; Ditchfield, R.; Pople, J. A. J. Chem. Phys. 1972, 56, 2257. (b) Francl, M. M.; Pietro, W. J.; Hehre, W. J.; Binkley, J. S.; DeFrees, D. J.; Pople, J. A.; Gordon, M. S. J. Chem. Phys. 1982, 77, 3654. (c) Clark, T.; Chandrasekhar, J.; Spitznagel, G. W.; Schleyer, P. von R. J. Comp. Chem. 1983, 4, 294.

${ }^{9}$ Frisch, M. J.; Trucks, G. W.; Schlegel, H. B.; Scuseria, G. E.; Robb, M. A.; Cheeseman, J. R.; Montgomery, J. A. Jr.; Vreven, T.; Kudin, K. N.; Burant, J. C.; Millam, J. M.; Iyengar, S. S.; Tomasi, J.; Barone, V.; Mennucci, B.; Cossi, M.; Scalmani, G.; Rega, N.; Petersson, G. A.; Nakatsuji, H.; Hada, M.; Ehara, M.; Toyota, K.; Fukuda, R.; Hasegawa, J.; Ishida, M.; Nakajima, T.; Honda, Y.; Kitao, O.; Nakai, H.; Klene, M.; Li, X.; Knox, J. E.; Hratchian, H. P.; Cross, J. B.; Adamo, C.; Jaramillo, J.; Gomperts, R.; Stratmann, R. E.; Yazyev, O.; Austin, A. J.; Cammi, R.; Pomelli, C.; Ochterski, J. W.; Ayala, P. Y.; Morokuma, K.; Voth, G. A.; Salvador, P.; Dannenberg, J. J.; Zakrzewski, V. G.; Dapprich, S.; Daniels, A. D.; Strain, M. C.; Farkas, O.; Malick, D. K.; Rabuck, A. D.; Raghavachari, K.; Foresman, J. B.; Ortiz, J. V.; Cui, Q.; Baboul, A. G.; Clifford, S.; Cioslowski, J.; Stefanov, B. B.; Liu, G.; Liashenko, A.; Piskorz, P.; Komaromi, I.; Martin, R. L.; Fox, D. J.; Keith, T.; Al-Laham, M. A.; Peng, C. Y.; Nanayakkara, A.; Challacombe, M.; Gill, P. M. W.; Johnson, B.; Chen, W.; Wong, M. W.; Gonzalez, C.; Pople, J. A. Gaussian 03; Gaussian, Inc., Pittsburgh PA, 2003 
(a)

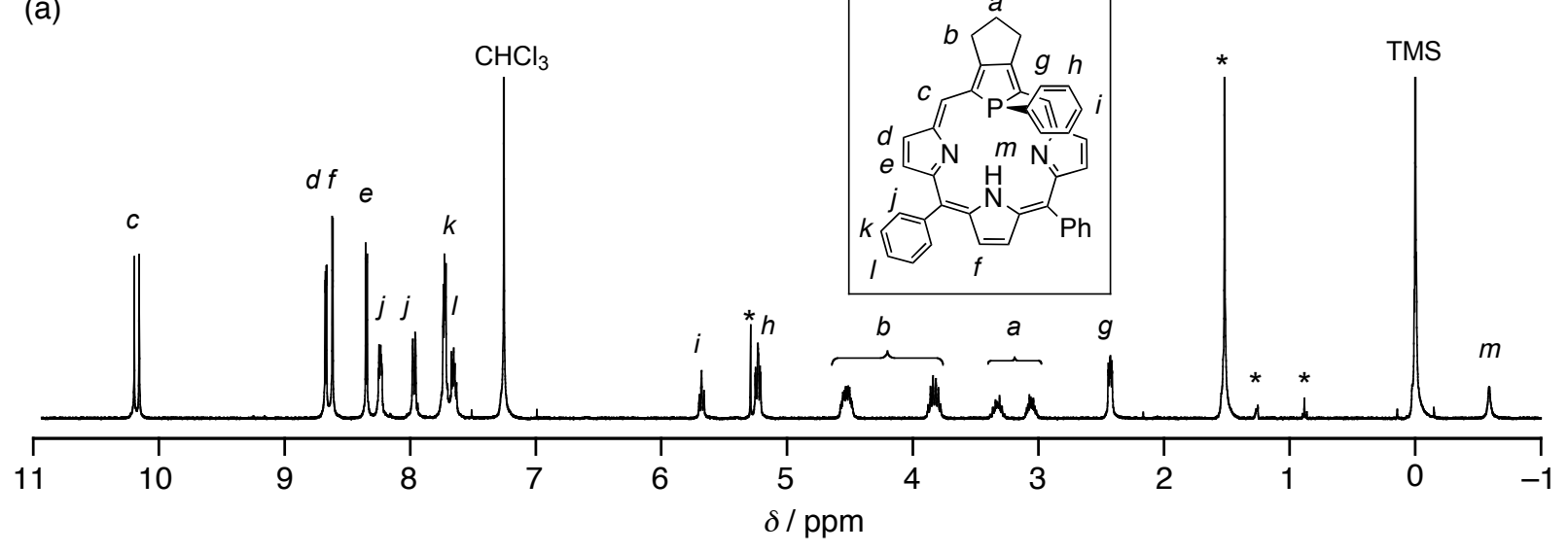

(b)

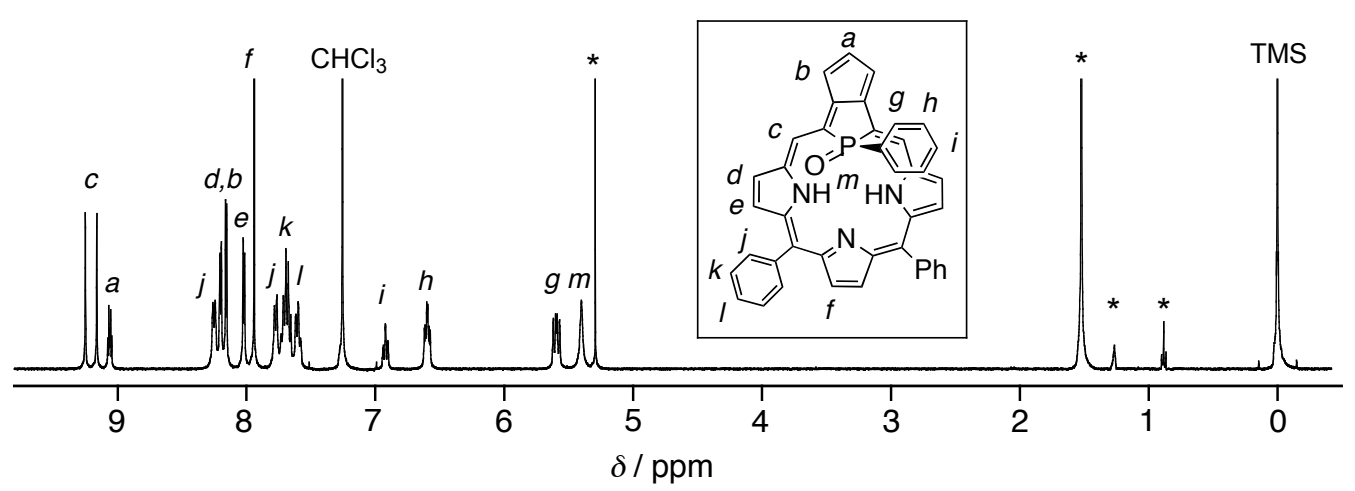

Figure S1. ${ }^{1} \mathrm{H}$ NMR spectra of (a) 7 and (b) 8 in $\mathrm{CDCl}_{3}$ (400 MHz). Asterisks (*) indicate the peaks of residual solvents and hydrocarbons.
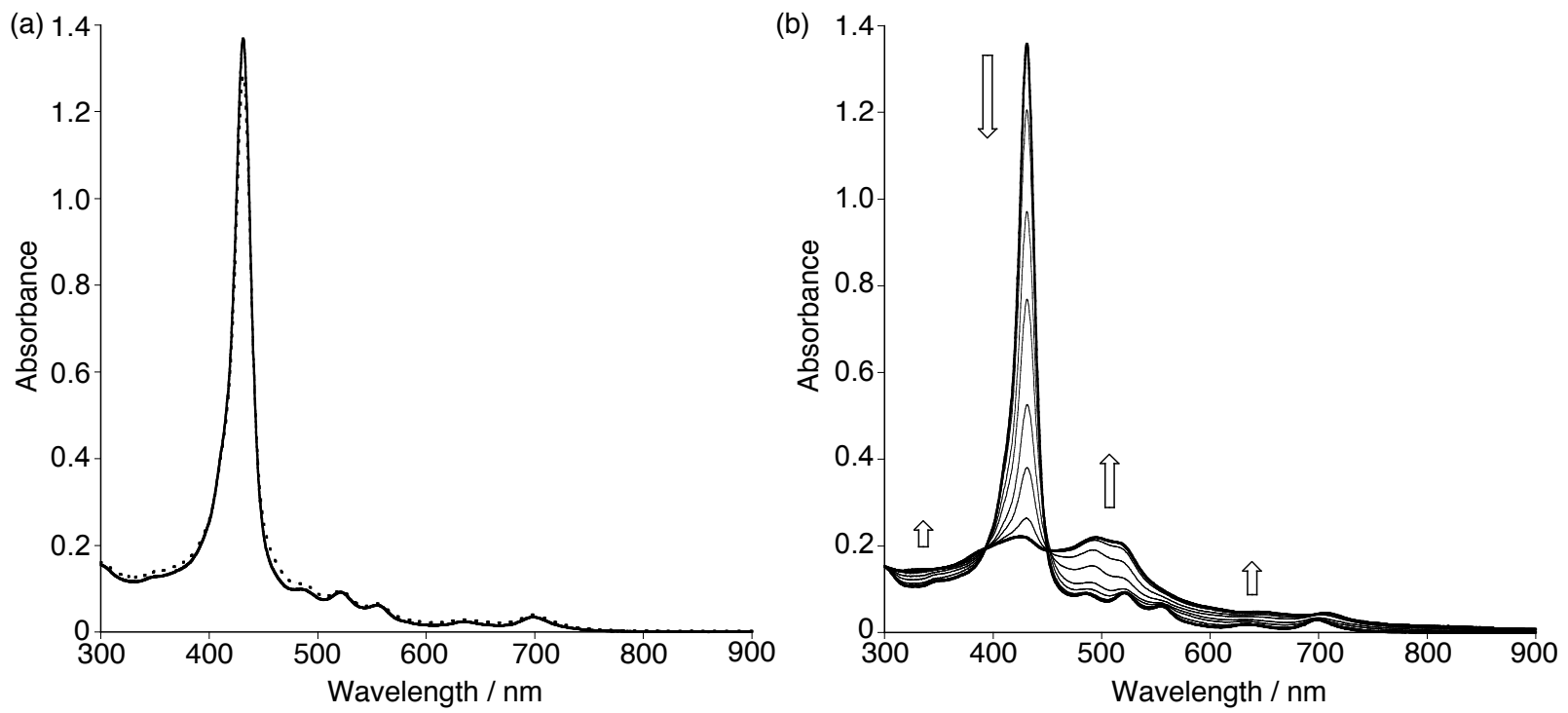

Figure S2. Time-dependence of the oxidative $\pi$-extension from $\mathbf{7}$ to $\mathbf{8}$, as monitored by UV-Vis absorption spectroscopy in $\mathrm{CH}_{2} \mathrm{Cl}_{2}$ at $25^{\circ} \mathrm{C}$. $[7]_{0}=1.0 \times 10^{-5} \mathrm{M}$. (a) In the dark (solid line, $0 \mathrm{~h}$; dotted line, $24 \mathrm{~h})$; (b) under normal light (0 12 h). 
(a)

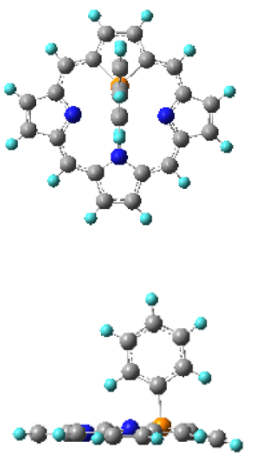

(b)

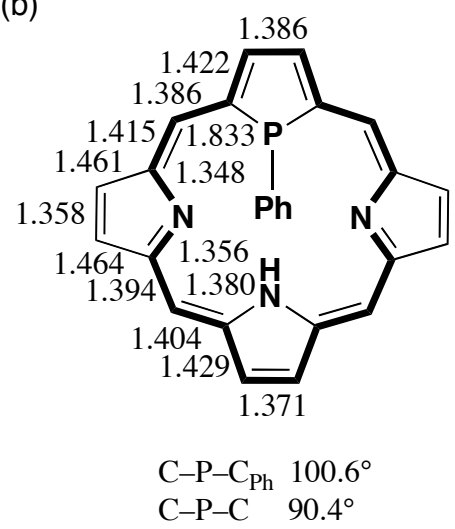

(c)
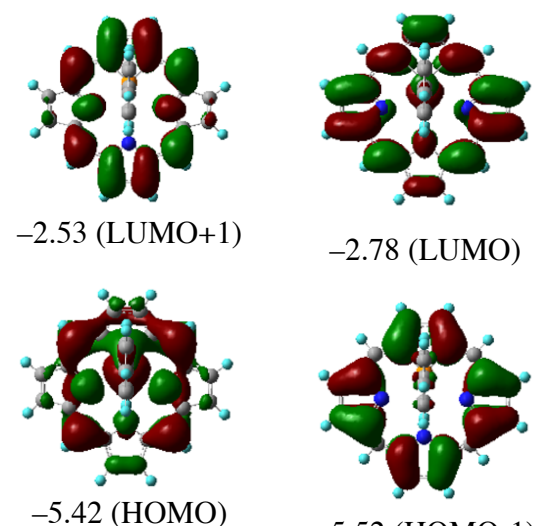

$-2.78($ LUMO)

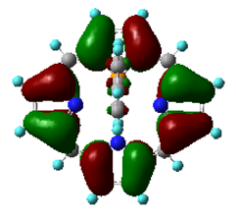

-5.52 (HOMO-1)

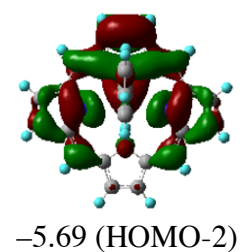

-5.69 (HOMO-2)

Figure S3. The results of DFT calculations for model compound 11. (a) Optimized structure: top (upper) and side (lower) views. The $\mathrm{C}, \mathrm{H}, \mathrm{N}$, and $\mathrm{P}$ atoms are indicated as gray, light blue, blue, and orange balls, respectively. (b) Selected bond lengths ( $\AA$ ) and bond angles (deg). (c) Molecular orbitals near HOMO and LUMO (orbital energies in eV).

(a)

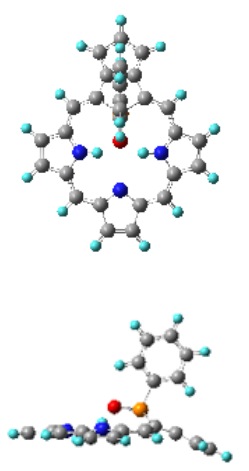

(b)

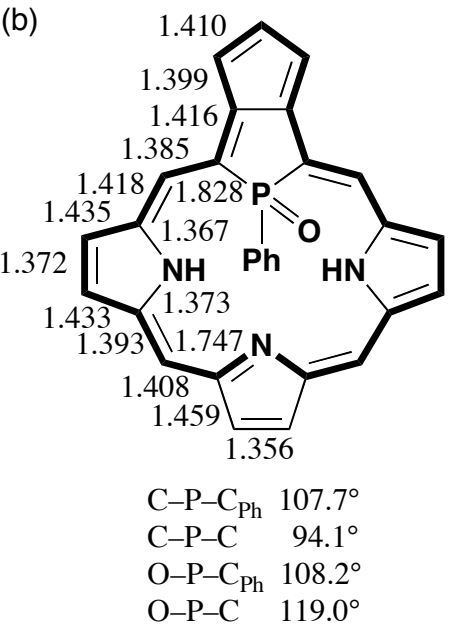

(c)

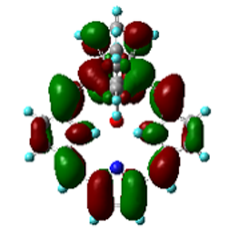

-2.37 (LUMO+1)

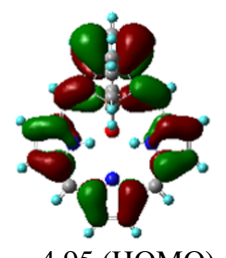

-4.95 (HOMO)

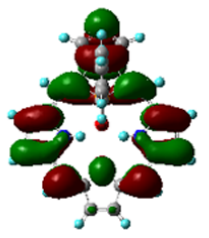

-3.02 (LUMO)

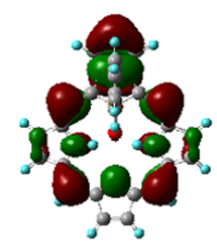

-5.28 (HOMO-1)

Figure S4. The results of DFT calculations for model compound 12. (a) Optimized structure: top (upper) and side (lower) views. The $\mathrm{C}, \mathrm{H}, \mathrm{N}$, and $\mathrm{P}$ atoms are indicated as gray, light blue, blue, and orange balls, respectively. (b) Selected bond lengths ( $\AA$ ) and bond angles (deg). (c) Molecular orbitals near HOMO and LUMO (orbital energies in $\mathrm{eV}$ ). 

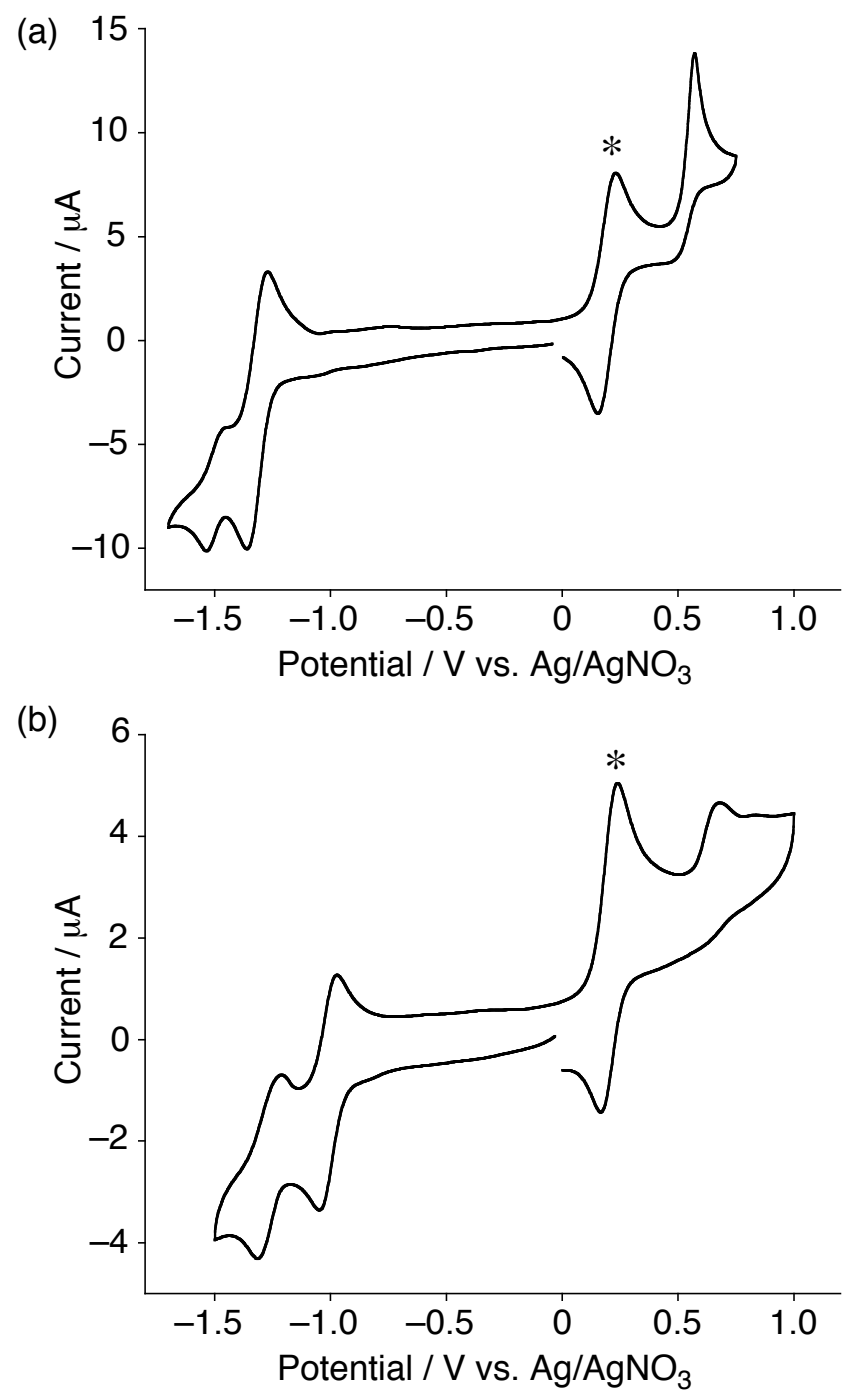

Figure S5. Cyclic voltammograms of (a) 7 and (b) 8. $0.1 \mathrm{M} n \mathrm{Bu}_{4} \mathrm{NPF}_{6}, \mathrm{Ag} / \mathrm{Ag}^{+}\left(0.01 \mathrm{M} \mathrm{AgNO}_{3}\right)$. Scan rate $20 \mathrm{mV} \mathrm{s}^{-1}$. Asterisk (*) indicates $\mathrm{Fc} / \mathrm{Fc}^{+}$couple. 

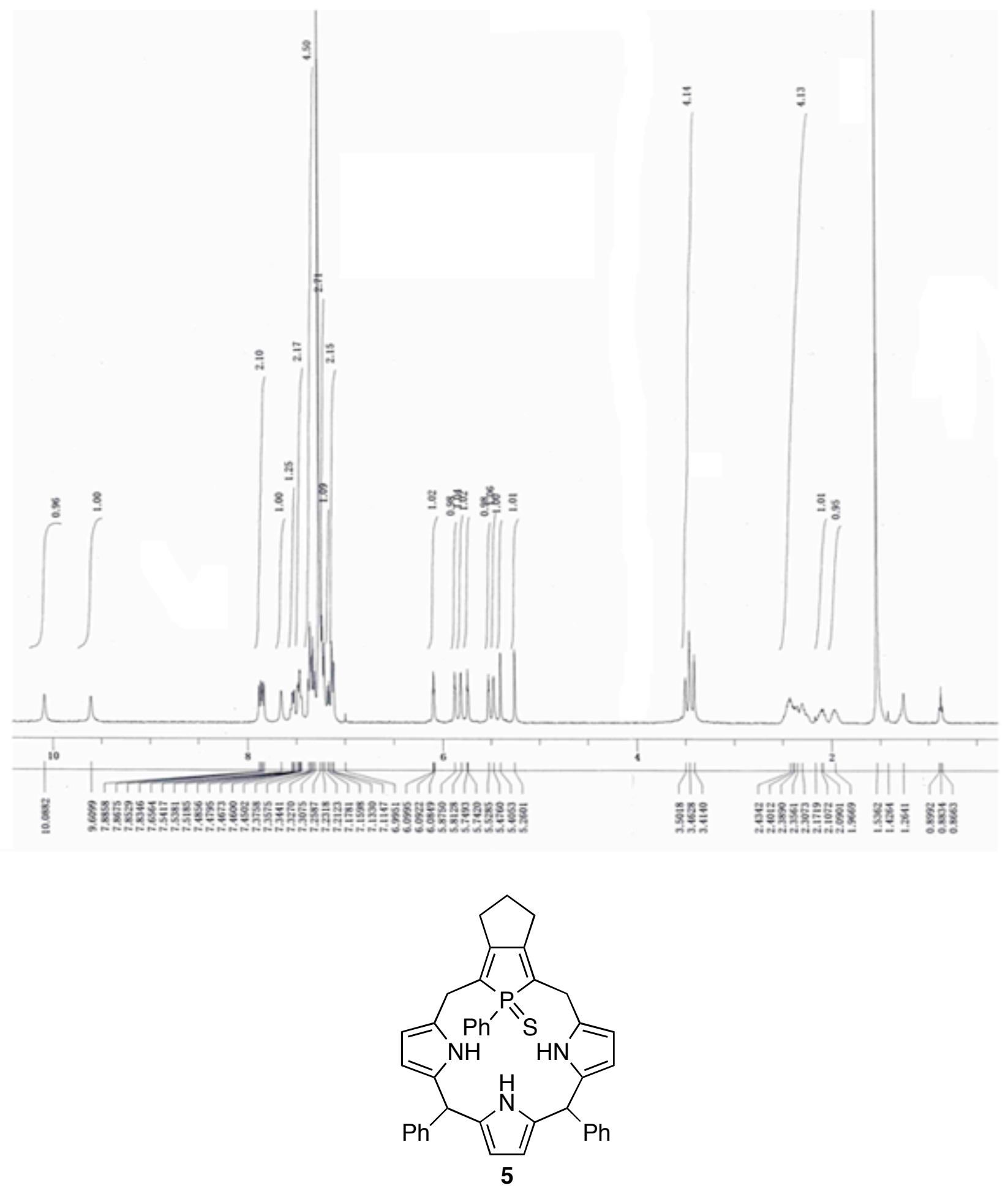

Figure S6. ${ }^{1} \mathrm{H}$ NMR spectrum of 5 in $\mathrm{CDCl}_{3}$. 


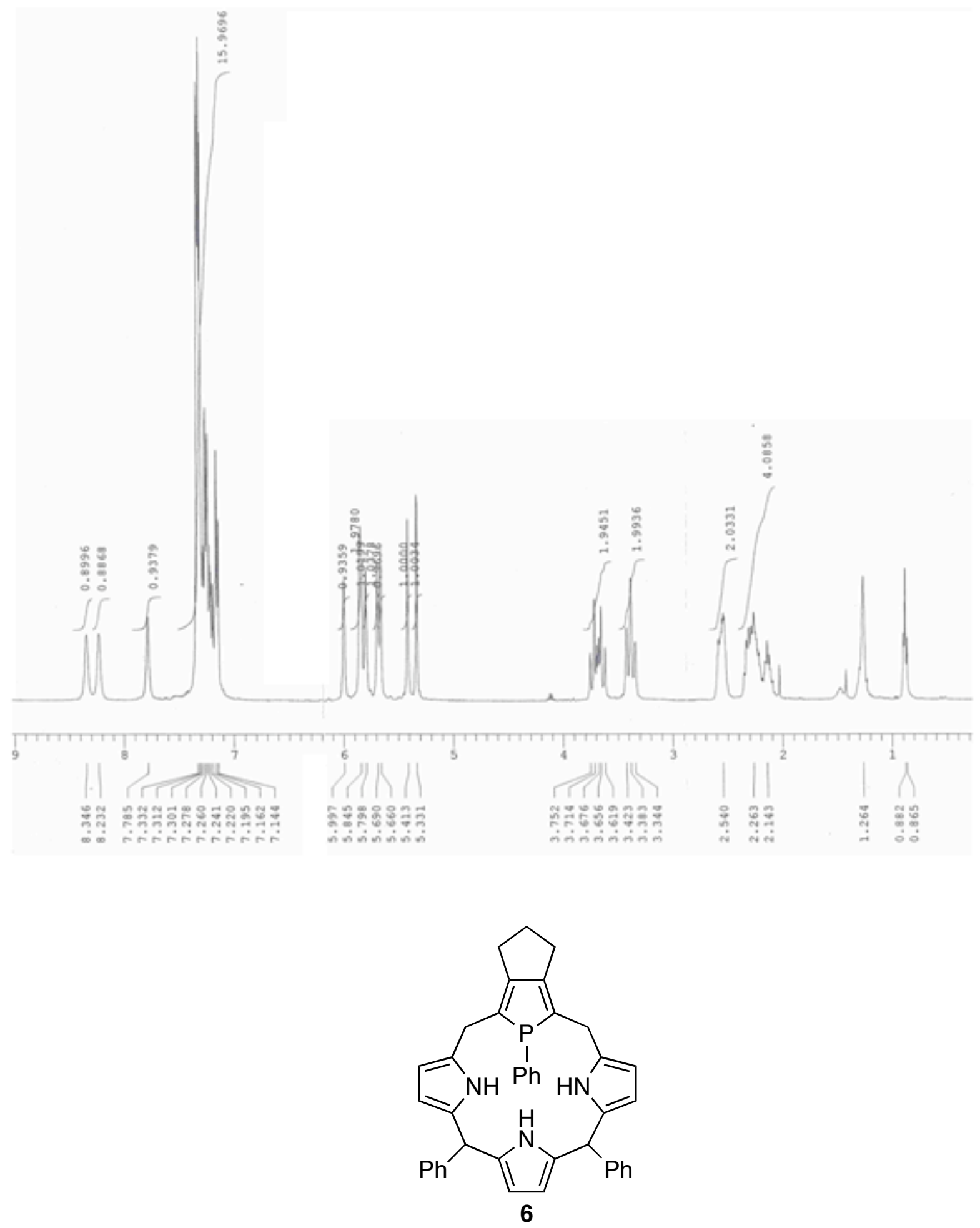

Figure S7. ${ }^{1} \mathrm{H}$ NMR spectrum of $\mathbf{6}$ in $\mathrm{CDCl}_{3}$. 

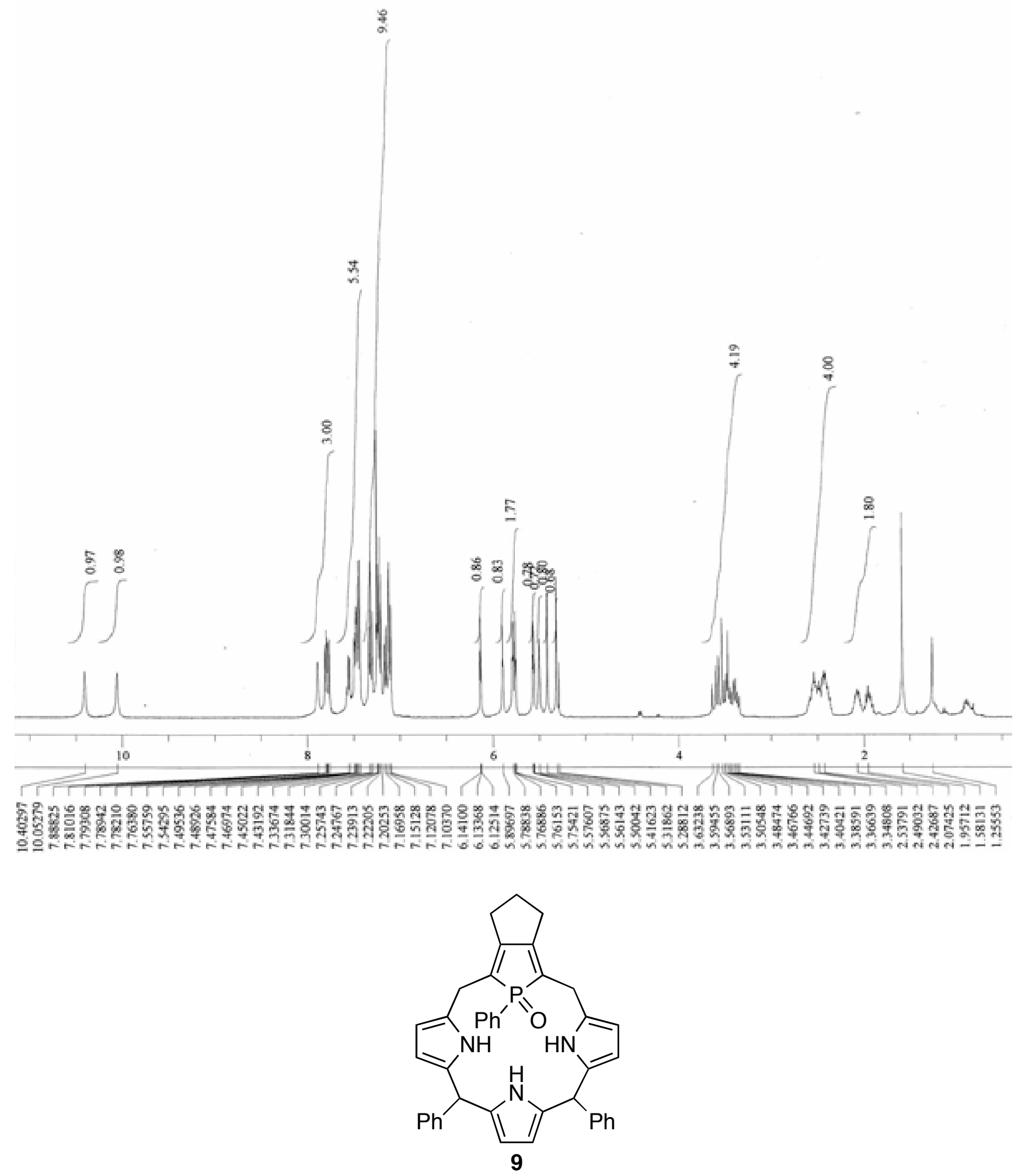

Figure S8. ${ }^{1} \mathrm{H}$ NMR spectrum of 9 in $\mathrm{CDCl}_{3}$. 

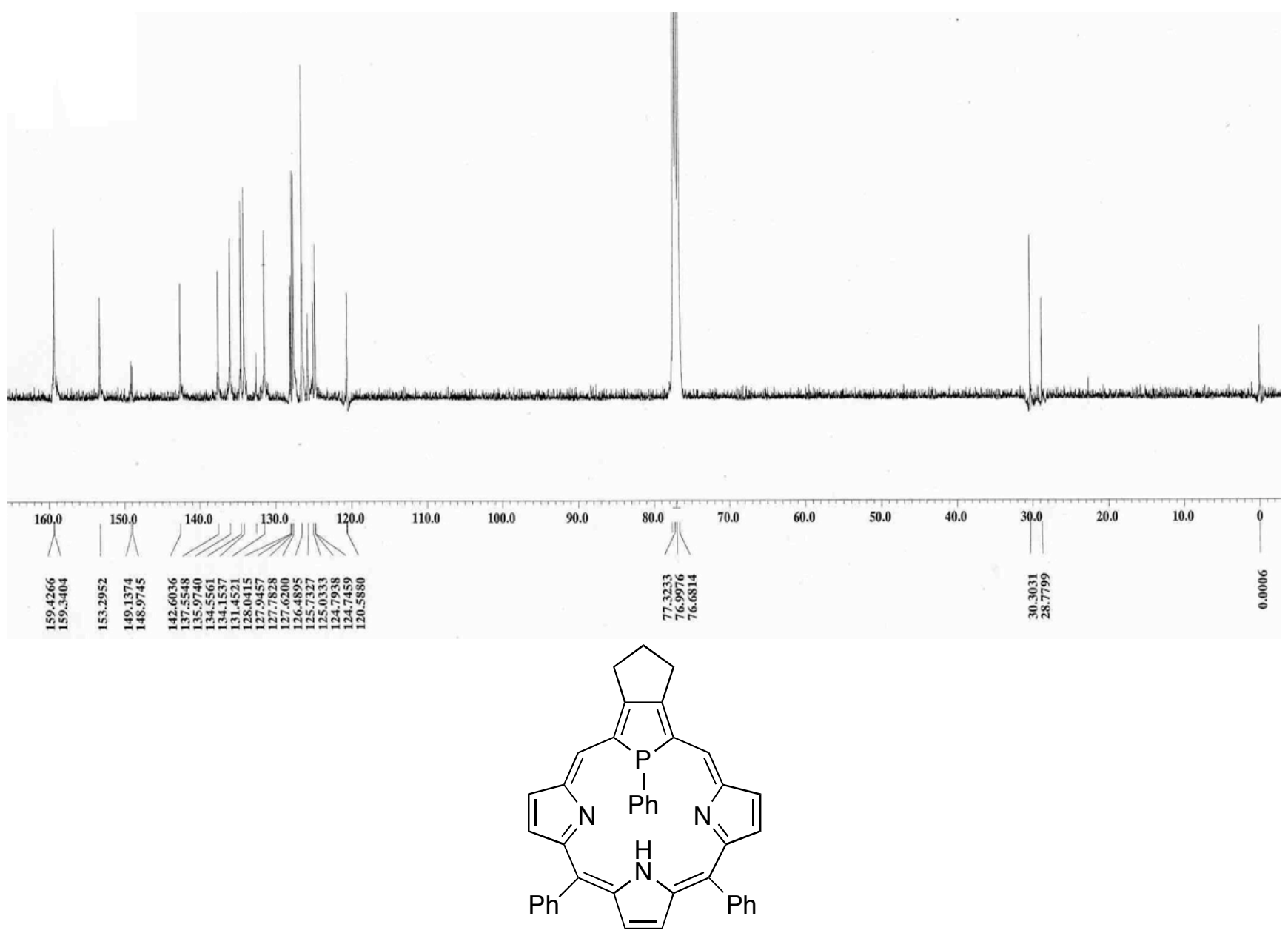

Figure S9. ${ }^{13} \mathrm{C}$ NMR spectrum of 7 in $\mathrm{CDCl}_{3}$. 


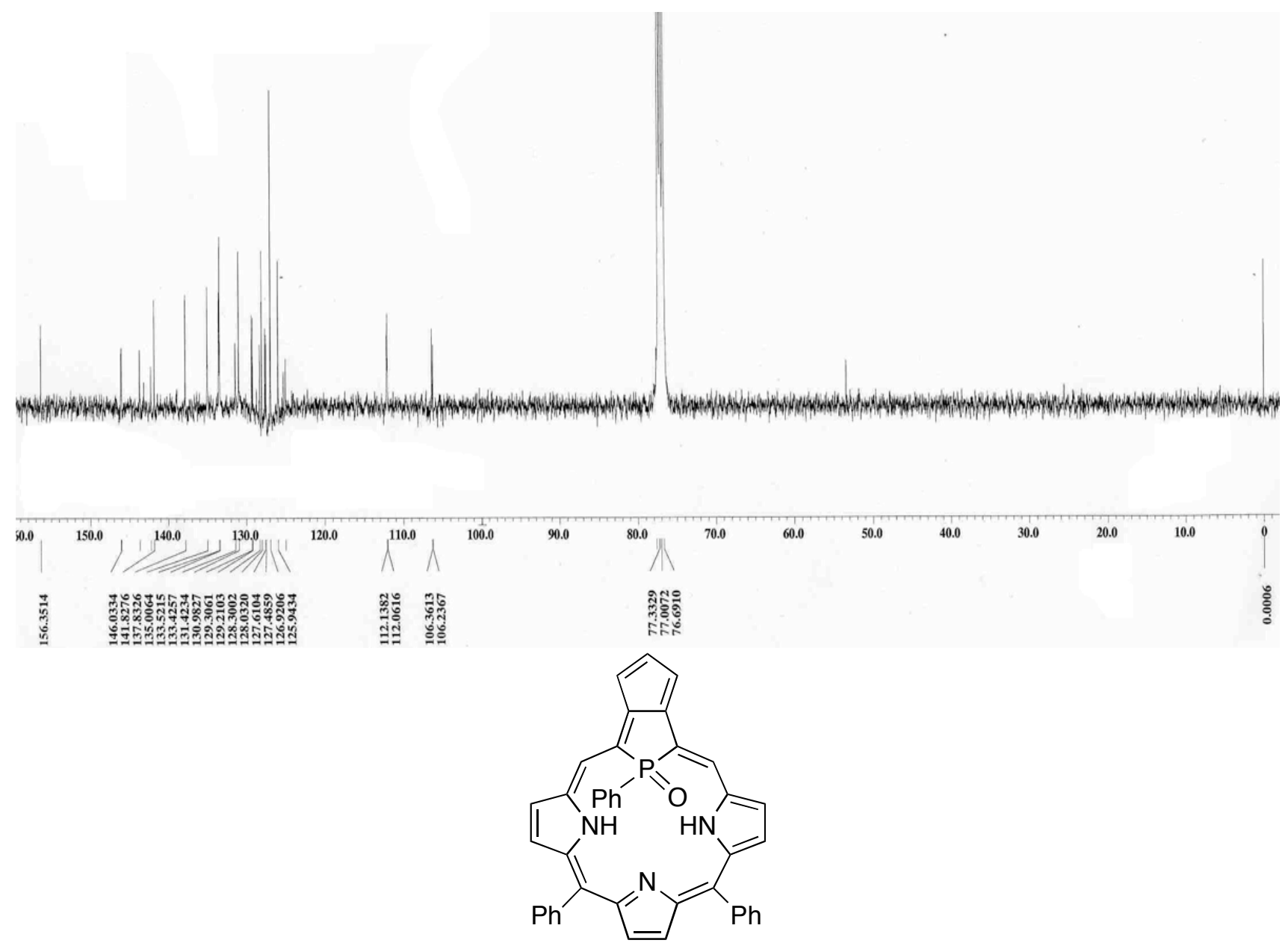

Figure S10. ${ }^{13} \mathrm{C}$ NMR spectrum of 8 in $\mathrm{CDCl}_{3}$. 